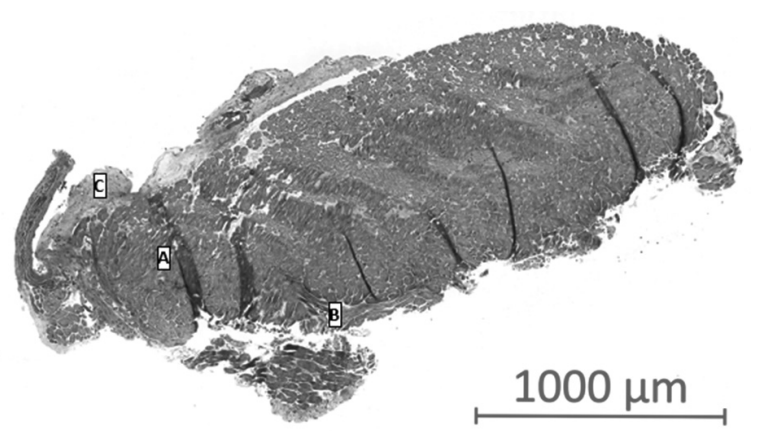

Abstract 125 Figure 1 Scanned Massons Trichrome biopsy from a NYHA class I patient, demonstrating typical examples of $(A)$ diffuse interstitial fibrosis, (B) replacement fibrosis and (C) endocardial thickening in a NYHA class I patient.


Abstract 125 Figure 2 Relationship between CVF and myofiber cross sectional area between controls and quartiles of MLHFQ.

samples were obtained from autopsies of 8 subjects who died of non-cardiac causes without signs of macroscopic or microscopic cardiac lesions.

Due to feasibility, $237 \mathrm{LV}$ biopsies were collected from 85 patients. Mean collagen volume fraction (CVFmean) was elevated in MR patients compared to controls $(16.1 \pm 10.1 \%$ vs, $4.3 \pm 2.7 \%, \mathrm{P}=0.002$ ), with significant $\mathrm{CVF}$ burden present even in the asymptomatic cohort $(14.7 \pm 9.2 \%)$. Pattern of fibrosis varied between diffuse interstitial fibrosis, coarse replacement fibrosis and endocardial thickening within and between each individual biopsy sample.

Histological quantification of muscle hypertrophy demonstrated an increase in myofiber cross-sectional area in MR patients compared to controls but did not differ between asymptomatic and symptomatic patients.

There was no relationship between CVF and age, gender, ventricular volumes, measures of MR severity, LVEF or extracellular volume (ECV). Late gadolinium enhancement, present in $34 \%$ of patients, was not predictive of higher CVFmean $(15.3 \pm 9.0 \%$ vs $16.5 \pm 10.8 \%, \mathrm{P}=0.63)$. CVFmean was higher in patients with Barlow's disease compared to FED (14 $\pm 8 \%$ vs
$10 \pm 11 \%, P=0.029)$ despite no difference in LV volumes, LVEF and MR severity.

Symptom burden was associated with the extent of fibrosis (MLHFQ and CVFmean, $\mathrm{R}=0.25, \mathrm{P}=0.03$ ), and ECV $(\mathrm{R}=0.19, \mathrm{P}=0.06)$. In a multivariable regression model, increasing CVFmean and ECV both independently predicted more advanced symptoms.

In summary patients with chronic primary degenerative MR myocardial fibrosis and myofiber hypertrophy increased when still asymptomatic. t. With symptomatic MR there were further increases in CVF and ECV expansion, without continued muscle hypertrophy, suggesting that an imbalance in the fibrosis:muscle ratio may be contributory to the development of symptoms. See Figure 1 and Figure 2.

Conflict of Interest None

\section{URINARY DESMOSINE, A BIOMARKER OF ELASTIN DEGRADATION IS SIGNIFICANTLY ELEVATED AND ASSOCIATED WITH MAXIMUM AORTIC ROOT SIZE AND AORTIC Z-SCORES IN PATIENTS WITH BICUSPID AORTIC VALVE}

'Zaid Iskandar*, ${ }^{2}$ Jeffrey Huang, ${ }^{3}$ Lynn Miller, ${ }^{4}$ Calvin Chin, ${ }^{2}$ Ify Mordi, ${ }^{5}$ Catherine Mcwilliam, ${ }^{5}$ David Goudie, ${ }^{5}$ Jonathan Berg, ${ }^{2}$ Alex Neagoie, ${ }^{2}$ Chim Lang, ${ }^{2}$ AnnaMaria Choy. 'Division of Molecular and Clinical Medicine; ${ }^{2}$ University of Dundee; ${ }^{3}$ NHS Fife; ${ }^{4}$ National Heart Centre Singapore; ${ }^{5}$ HSS Tayside, Dundee

\subsection{6/heartjnl-2019-BCS.123}

Introduction Bicuspid aortic valve (BAV) affects up to $2 \%$ of the general population and is associated with a significant risk of aortic dilatation and aortic dissection. Therefore, patients are normally routinely followed up with serial echocardiography. Aortic dilatation involves elastin breakdown as part of extracellular matrix degradation. Desmosine is a specific amino acid cross-link between mature elastin molecules and is released into plasma and urine following elastin degradation. Following on from previous pilot data in the DESMA study on patients with Marfan Syndrome which demonstrated a significant correlation between plasma desmosine and aortic root size, we explored whether this same relationship is seen in patients with other forms of aortopathies, specifically the BAV cohort.

Methods Urinary and plasma desmosine were measured in 20 patients with BAV and compared to age-matched healthy controls. Urinary desmosine (uDES) and plasma desmosine (pDES) levels were measured in the same laboratory using liquid chromatography-tandem mass spectrometry (LC-MS).

Results The cohort was predominantly male (75\%) and mean age was $49.3 \pm 17.3$ years old. All the patients had normal LV systolic function and 9 (45\%) patients had treated hypertension. Mean aortic root size was $35 \pm 5.8 \mathrm{~mm}$. Compared to controls, both uDES $(15.9 \pm 4.6$ vs $7.2 \pm 2.8 \mathrm{ng} / \mathrm{mg}$ creatinine, $\mathrm{p}<0.001)$ and pDES $(0.3 \pm 0.1$ vs $0.26 \pm 0.075 \mathrm{ng} / \mathrm{mL}$, $\mathrm{p}=0.01)$ were significantly elevated in patients with BAV. Urinary desmosine was significantly correlated (Figure 1) with aortic root size $(\mathrm{r}=0.65, \mathrm{p}=0.002)$, and aortic root $\mathrm{z}$-score $(r=0.59, p=0.007)$, however there was no significant correlation seen between plasma desmosine and aortic root size $(\mathrm{r}=0.42, \mathrm{p}=0.06)$ or aortic root $\mathrm{z}$-score $(\mathrm{r}=0.14, \mathrm{p}=0.56)$.

Conclusion Urinary and plasma desmosine levels are significantly higher in patients with BAV compared to controls. Urinary desmosine is also significantly associated with aortic root size and $\mathrm{z}$-score, reflecting higher elastin degradation in 


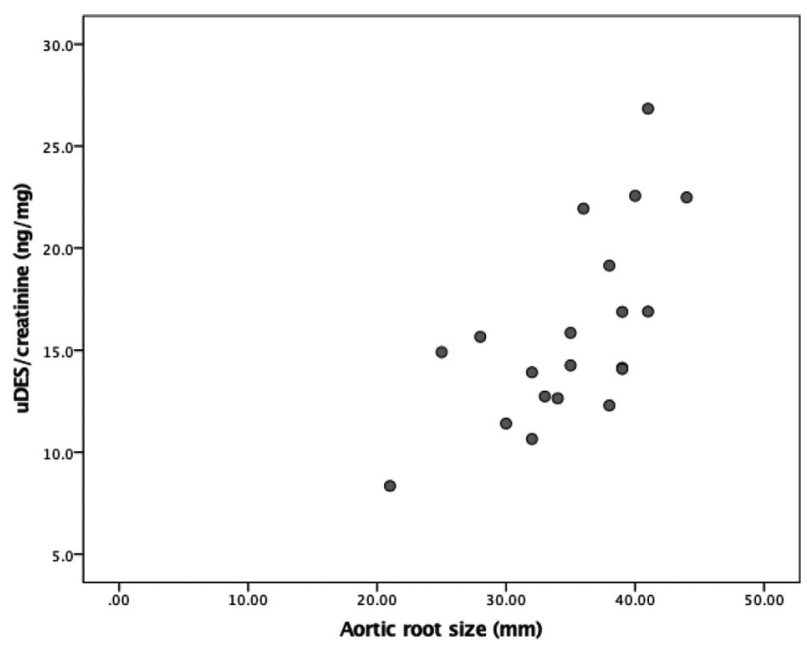

Abstract 126 Figure 1 Correlation between uDES (ng/mg creatinine) and aortic root size $(\mathrm{mm})$

patients with larger aortic roots. This suggests a potential utility of desmosine as a biomarker in patients with BAV. Larger studies are needed to test this hypothesis.

Conflict of Interest None

\section{DEVELOPMENTAL ROCK DOWNREGULATION DISRUPTS SARCOMERIC STRUCTURE RESULTING IN THE DEVELOPMENT OF HYPERTROPHIC CARDIOMYOPATHY}

${ }^{1}$ Kate Bailey*, ${ }^{2}$ Guy MacGowan, ${ }^{1}$ Simon Tual-Chalot, 'Lauren Phillips, ${ }^{3}$ Timothy J Mohun, ${ }^{1}$ Deborah J Henderson, ${ }^{1}$ Helen Arthur, ${ }^{1}$ Simon Bamforth, ${ }^{1}$ Helen M Phillips. ${ }^{1}$ Newcastle University; ${ }^{2}$ Freeman Hospital; ${ }^{3}$ Francis Crick Institute

\subsection{6/heartjnl-2019-BCS.124}

Introduction Congenital heart defects are common, affecting $\sim 1 \%$ of live births, while adult heart disease is the main cause of death in the UK. Defects acquired during foetal development can have a lasting detrimental effect on adult heart function. Therefore, understanding the underlying mechanisms involved in cardiac development and disease progression are of particular importance.

Cardiomyopathy is a disease of the heart muscle. Hypertrophic cardiomyopathy (HCM) is one of the most common forms of cardiomyopathy; mutations have been identified in the troponin complex, a key component of the sarcomere, signifying that disruption to sarcomeric integrity plays an important role in disease progression.

Rho Kinase (ROCK) is expressed in the heart during development and is known to be a regulator of actin-myosin contraction through the phosphorylation of the troponin complex. Our aim was to determine the effect of developmental ROCK downregulation on sarcomeric integrity and impact this had on the function of the adult heart.

Methods Cre-LoxP technology was utilised to create a conditional mouse model in which ROCK was specifically downregulated in the cardiomyocytes from E9.25, during cardiac development. Histological techniques were used to assess the embryonic and adult heart phenotype. Analysis of the cytoarchitecture was performed by TEM while cine cardiac MRI was used to assess overall adult heart function.

Results and Conclusions: Developmental downregulation of ROCK in the cardiomyocytes resulted in loss of sarcomere integrity at E10.5, associated with a reduction in the levels of phosphorylation of cardiac Troponin I and $\mathrm{T}$, and reduced cardiomyocyte proliferation at E11.5. This caused abnormal myocardial wall development, where the compact myocardium failed to thicken. The impact of these embryonic abnormalities, triggered compensatory foetal cardiomyocyte hypertrophy, which persisted throughout postnatal development and into adult life. Over time this continued hypertrophy became detrimental, triggering cardiac remodelling. Mutants exhibit key features of HCM including concentric hypertrophy with systolic dysfunction, fibrosis and re-expression of foetal genes. This data suggests a novel developmental origin of the sarcomeric phenotype of HCM and indicates disruption in ROCK signalling may contribute to the pathogenesis of HCM.

(Bailey et al., Disruption of embryonic ROCK signalling reproduces the sarcomeric phenotype of Hypertrophic Cardiomyopathy. JCI Insight, in press)

Conflict of Interest None

\section{THE IMPACT OF AORTIC VALVE REPLACEMENT ON SURVIVAL IN PATIENTS WITH NORMAL FLOW LOW GRADIENT SEVERE AORTIC STENOSIS: A PROPENSITY- MATCHED COMPARISON}

${ }^{1}$ Roxy Senior*, ${ }^{2}$ Sahrai Saeed, ${ }^{3}$ Anastasia Vamvakidou, ${ }^{4}$ Rajdeep Khattar, ${ }^{4}$ Wei Li. ${ }^{1}$ Royal Brompton Hospital and National Heart and Lung Institute, Imperial College, London; ${ }^{2}$ Haukeland University Hospital, Bergen, NO; ${ }^{3}$ Northwick Park Hospital; ${ }^{4}$ Royal Brompton Hospital

\subsection{6/heartjnl-2019-BCS.125}

Introduction To assess the survival benefit of aortic valve replacement (AVR) in patients with normal flow low gradient severe aortic stenosis (AS).

Methods A retrospective study of prospectively collected data of 276 patients (mean age $75 \pm 15$ years, 51\% male) with normal transaortic flow (flow rate [FR] $200 \mathrm{ml} / \mathrm{s}$ or stroke volume index [SVi] $35 \mathrm{ml} / \mathrm{m}^{2}$ ) and severe AS (aortic valve area [AVA] $<1.0 \mathrm{~cm}^{2}$. The outcome measure was all-cause mortality.

Results Of the 276 patients, 55\% $(\mathrm{n}=151)$ were medically treated while $45 \%(n=125)$ underwent an AVR. Over a mean follow-up of $3.2 \pm 1.8$ years (range 0-6.9 years) a total of $96(34.8 \%)$ deaths occurred: $17(13.6 \%)$ in AVR group versus $79(52.3 \%)$ in those medically treated, when transaortic flow was defined by FR $(\mathrm{p}<0.001)$ (figure 1$)$. When transaortic flow was defined by SVi, a total of $79(31.3 \%)$ deaths occurred: $18(15.1 \%)$ in AVR group versus 61 $(45.9 \%)$ in medically treated $(\mathrm{p}<0.001)$. In a propensitymatched multivariable Cox regression analysis adjusting for age, gender, body surface area, smoking, hypertension, diabetes mellitus, atrial fibrillation, peripheral vascular disease, chronic kidney disease, left ventricular ejection fraction, left ventricular mass and mean aortic gradient, not having AVR was associated with a 6.3 fold higher HR of all-cause mortality (HR 6.28; 95\% CI 3.34-13.16, p<0.001) when flow was defined by FR. In the SVi-guided model it was 3.83 fold (HR 3.83; 95\% CI 2.30-6.37, p<0.001).

Conclusion In patients with normal flow low gradient severe AS, AVR was associated with a significantly improved survival compared to those who received standard medical treatment. Conflict of Interest No 